\title{
Doppler ultrasound in the estimation of the severity of pulmonary infundibular stenosis in infants and children
}

\author{
A B HOUSTON, I A SIMPSON, C D SHELDON, W B DOIG, E N COLEMAN \\ From the Department of Cardiology and University Department of Child Health, Royal Hospital for Sick \\ Children, Glasgow
}

SUMMARY Pressure gradients estimated by Doppler echocardiography were compared with values obtained at cardiac catheterisation in 31 children (aged seven days to 16 years, mean 2 years 7 months) with pulmonary infundibular stenosis including 16 with tetralogy of Fallot. Various parasternal and subcostal positions were explored to obtain the maximum velocity of blood flow and the obstructive gradient was calculated from the modified Bernoulli formula. The gradient across the obstruction could be measured directly at the time of catheterisation in only 21 patients. The correlation coefficient for the Doppler and total measured gradients was $r=0.90$ for catheter entry and $r=0.77$ for catheter withdrawal. Doppler ultrasound, by measuring the total gradient from the right ventricle to the pulmonary artery, provides a non-invasive assessment of the severity of pulmonary stenosis, and in those with infundibular obstruction allowance need not be made for possible energy losses caused by the elongated obstruction or the presence of narrowing at more than one level.

The development of Doppler echocardiography as a technique to provide a non-invasive assessment of the pressure gradient in obstructive cardiac lesions ${ }^{12}$ has been an important advance in the management of congenital heart disease. Several studies attest to its accuracy in pulmonary valve stenosis $^{3-10}$ but Doppler measurements have been compared with measured gradients in only two patients with infundibular stenosis. ${ }^{5}$ With Doppler echocardiography the pressure gradient is calculated by measuring the maximum velocity of blood flow across the obstruction and applying the modified Bernoulli equation. This simplified equation has generally been applied where there is a discrete obstruction and ignores energy losses due to friction. There may therefore be potential errors in applying the formula to patients with infundibular pulmonary stenosis where the obstruction can be elongated or can occur at more than one level. We therefore considered it important to review our

Requests for reprints to Dr A Houston, Royal Hospital for Sick Children, Yorkhill, Glasgow G38SJ.

Accepted for publicaton 13 January 1986 experience and assess the validity of the modified Bernoulli formula in the measurement of pressure gradient in patients with infundibular pulmonary stenosis.

\section{Patients and methods}

We studied 31 infants and children aged seven days to 16 years (mean 2 years 7 months) with infundibular pulmonary stenosis proved by cardiac catheterisation and angiocardiography. Fifteen were considered to have infundibular and possible valve stenosis with a ventricular septal defect and 16 had tetralogy of Fallot. One had undergone repair of tetralogy of Fallot, and although the angiocardiogram suggested some residual infundibular narrowing the pressure gradient was small $(15 \mathrm{~mm}$ $\mathrm{Hg}$ ). At catheterisation pressures were measured through a fluid filled catheter and the gradient across the obstruction was recorded as the peak to peak difference not only on catheter withdrawal but also as the catheter was initially advanced into the right ventricle and pulmonary artery. Such measurements were possible in only 21 patients (11 had ventricular septal defect with infundibular pulmonary stenosis 
and 10 had tetralogy of Fallot); in the others no attempt was made to pass the catheter into the pulmonary artery because of the severity of the obstruction or the attempt was abandoned when arrhythmia developed. The pressure recordings were reviewed and the measurement of the peak to peak difference was made independently by an observer who was unaware of the Doppler findings.

Where possible an effort was made to obtain a Doppler recording before the child was sedated for catheterisation. In several younger ones this was not possible since even minor movements of the child made it impossible to obtain the exact angulation of the probe or to maintain it for sufficiently long to record an adequate Doppler signal. With the child quiet, sedated, or asleep, satisfactory recordings were obtained, however, from all patients. Of the 21 in whom the gradient was measured 11 underwent the Doppler study while sedated for catheterisation, three within 24 hours of catheterisation, and the other seven on admission for subsequent operation. In one patient the Doppler study was performed with simultaneous measurement of pressure in the pulmonary artery and right ventricle by two catheters. In the other patients the Doppler study was not carried out with a catheter in the pulmonary artery because of possible complications related to infundibular obstruction.

The Doppler equipment consisted of a multifrequency continuous and pulsed wave unit (Alfred, Vingmed) which was used in the continuous mode with a $2 \mathrm{MHz}$ transducer, the signal being monitored on a spectrum analyser (Doptek) fitted with a movable marker line. As the examination proceeded the line was, as necessary, moved to the average of the highest velocities obtained; this permitted easy comparison of the received frequency shift with the previous maximum and ensured that the highest value for the whole investigation was obtained. A cross sectional echocardiogram was initially obtained with an ATL sector scanner, which confirmed the diagnosis and, by demonstrating the intracardiac anatomy, suggested positions from which the Doppler beam might most accurately be aligned along the right ventricular outflow. Although this position was initially used, other subcostal, precordial, suprasternal, and supraclavicular sites were explored to obtain the signal with the maximum Doppler shift. Care was taken to obtain reproducible signals showing as far as possible many high velocity signals with a clear and complete envelope. At the end of the examination the level of the marker line on the spectrum analyser indicating the maximum frequency shift (in $\mathrm{KHz}$ ) for the whole examination was used to calculate the maximum velocity $(V)$ in metres per second and the pres- sure gradient $(P)$ in $\mathrm{mm} \mathrm{Hg}$ from the modified Bernoulli formula, $P=4 \mathrm{~V}^{2}$. The estimated and measured pressure gradients were compared in the 21 patients in whom both were measured.

\section{Results}

Pressure gradients measured at catheterisation from separate right ventricular and pulmonary arterial recordings as the catheter was advanced were compared with those obtained on catheter withdrawal. There was considerable variation with a correlation coefficient of $0.87(y=0.92 x+6 \cdot 6)$. The difference was generally related to variation in the right ventricular pressure measurement on catheter withdrawal caused by extrasystoles which altered the right ventricular pressure values making it difficult for the independent observer to assess a true right ventricular systolic pressure.

Satisfactory Doppler recordings were obtained

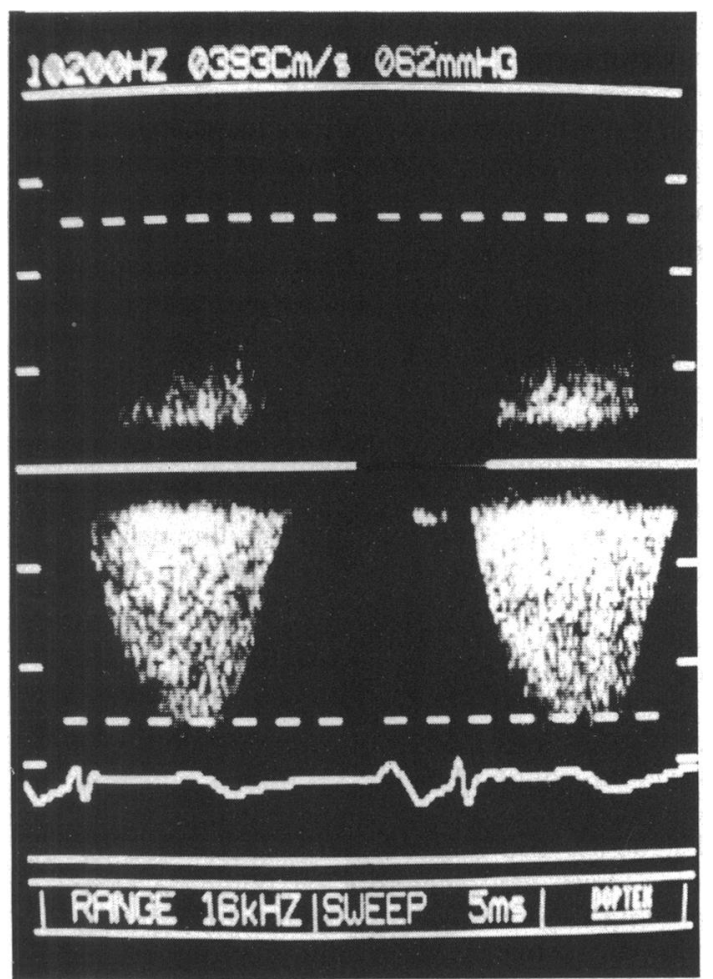

Fig. 1 Recording of the Doppler signal from the spectrum analyser from a patient with infundibular pulmonary stenosis. The signal which is systolic in timing shows a negative deflection indicating flow away from the transducer. The broken lines are the markers which give a maximum frequency shift of $10200 \mathrm{Kz}$ representing a velocity of $3.93 \mathrm{~ms}^{-1}$ and a gradient of $62 \mathrm{~mm} \mathrm{Hg}$. 


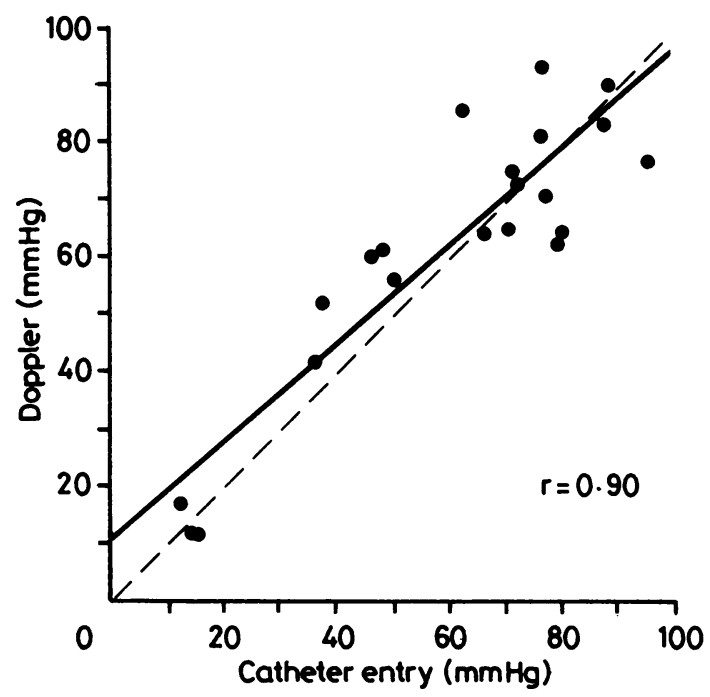

Fig. 2 Comparison of the pressure gradients measured at catheter entry and estimated by Doppler signal. The linear regression line has a gradient of $0.85(y=0.85 x+10 \cdot 70)$.

from all patients (Fig. 1). Maximum blood flow velocities were obtained almost equally frequently from subcostal and parasternal positions as flow away from the transducer. In one patient a satisfactory signal could be obtained from a parasternal position only from the second left intercostal space as flow towards the transducer. Comparison of the maximum Doppler gradient with that measured on catheter withdrawal showed a correlation coefficient of $0.77(y=0.77 x+17.05)$; for catheter entry a correlation coefficient of 0.90 was obtained (Fig. 2). Since the Doppler study was performed with the child in a relatively undisturbed state and not at catheter withdrawal, the most appropriate comparison is that of Doppler with pressure gradient at entry.

In the patient in whom two catheters were used the peak to peak gradient was $62 \mathrm{~mm} \mathrm{Hg}$ on entry, $70 \mathrm{~mm} \mathrm{Hg}$ on withdrawal, and $80 \mathrm{~mm} \mathrm{Hg}$ with two catheters (instantaneous maximum $85 \mathrm{~mm} \mathrm{Hg}$ ) and the simultaneous Doppler value was $86 \mathrm{~mm} \mathrm{Hg}$.

\section{Discussion}

The ability of Doppler ultrasound to provide an assessment of the pressure gradient in patients with pulmonary valve stenosis has been confirmed by several groups of investigators. Studies have shown a good correlation between the gradient measured at catheterisation and that calculated from Doppler ultrasound, both continuous and pulsed, in patients with discrete pulmonary valve stenosis. ${ }^{3-10}$ Reports on Doppler ultrasound in infundibular stenosis are scanty $^{35}$ and measured pressures were given for only two patients. ${ }^{5}$

The Bernoulli formula relates the pressure gradient to the kinetic energy content of a fluid before and after an obstruction and this will not change whether the obstruction is discrete or elongated. Application of the modified formula $P=4 V^{2}$, however, assumes that there is no energy loss due to viscous friction which may become more important as the length of the obstruction increases. It has not yet been confirmed that this formula can be applied where the narrowed segment is elongated or curved, or when obstruction occurs at more than one level, as can happen in patients with tetralogy of Fallot or with infundibular and valve stenosis. Thus we considered it appropriate to examine whether under these circumstances the simple modified Bernoulli formula remained valid or whether allowance needed to be made for frictional losses or obstruction at more than one level.

One of the major difficulties in any study of Doppler assessment of gradient is accurate measurement of the method against which it is to be compared. The correlation is better when the Doppler measurement is made at catheterisation rather than at another time ${ }^{811}$ and is further improved by the simultaneous measurement of the gradient with two catheters, one proximal and the other distal to the obstruction. Even then Doppler gives the maximum instantaneous pressure drop and not the peak to peak difference. ${ }^{89}$ This may be important in aortic stenosis ${ }^{912}$ but, because of the low pulmonary artery pressure, it is of less importance in pulmonary stenosis and for practical clinical purposes can be ignored. Although Doppler echocardiography was performed at the time of catheterisation in 11 patients in this study, in all but one patient it was considered inappropriate to do so at catheter withdrawal or with two catheters because of possible complications related to leaving the catheter through the narrowed infundibulum while the Doppler investigation was undertaken. In the patient in whom two catheters were used the results confirmed that correlation was improved when the Doppler result was compared with the result measured by this technique.

We found that the apparent variation in pressure gradient with time when entry and withdrawal pressures were compared with Doppler was associated with difficulties in obtaining an accurate right ventricular pressure after withdrawal in some patients in whom extrasystoles occurred. Thus it seems more appropriate to compare the Doppler measurement performed when the child was undisturbed with those obtained as the catheter entered the right ven- 
tricle (and when there were no extrasystoles) and when a stable pulmonary artery pressure was obtained. This does provide the better correlation and suggests that the simple modified Bernoulli formula is as applicable in cases with infundibular stenosis or infundibular and valve stenosis as in those with discrete obstruction. In keeping with previously reported findings, ${ }^{35}$ however, Doppler has not been shown to give any information on the level of obstruction or the relative contribution of infundibular or valvar narrowing to the total gradient. Using pulsed wave Doppler Hatle and Angelsen have been able to predict the presence of infundibular stenosis, ${ }^{3}$ but the equipment that they used could not measure the high velocities occurring when obstruction was clinically important. Pulsed wave Doppler with a lower transducer frequency or higher pulse repetition frequency can measure higher velocities from areas near to the transducer but neither pulsed nor continuous Doppler has yet been shown to be able to determine the relative importance of infundibular and valvar obstruction in these cases.

Doppler ultrasound has been able to provide an assessment of the pressure gradient when this could not be measured because of the potential hazard of passing a catheter through a severely narrowed outflow tract. In some of these patients clinical and echocardiographic assessment indicate the need for angiocardiography or operation but there are others in whom the degree of infundibular stenosis changes with time and a non-invasive technique for performing serial measurement of the obstruction will be important. Although Doppler ultrasound cannot yet determine the relative importance of valve or subvalve stenosis the decision on the need for operation is based on the total gradient and anatomical knowledge (echocardiographic or angiocardiographic) of the right ventricular outflow tract is much more useful to the surgeon than physiological information about the relative contributions of the infundibulum and valve to the total obstruction.

Those who are unfamiliar with the principles of the Bernoulli formula may be surprised that it provides accurate values in patients, such as those with tetralogy of Fallot, in whom there can be considerable variation with time in the severity of the infundibular stenosis with major changes in the volume of blood flowing through the right ventricular outflow. In these patients, however, the pulmonary artery pressure is relatively constant and the right ventricular pressure, which is at systemic level will show only relatively small changes; and although variation in flow can occur the pressure gradient will change little. The Bernoulli formula measures the pressure drop that is related to the increase in veloc- ity and is independent of size of orifice and can therefore provide an accurate prediction of the gradient.

The results of this study are in accord with those previously reported in patients with discrete obstruction and the two with infundibular stenosis. They indicate that allowance need not be made for energy losses due to friction, and that the simple modified Bernoulli formula can provide an accurate assessment of the severity of pulmonary stenosis whether it occurs at valve or infundibular level or at both.

\section{References}

1 Hatle L, Brubakk A, Tromsdal A, Angelsen B. Noninvasive assessment of pressure drop in mitral stenosis by Doppler ultrasound. Br Heart f 1978; 40: 131-40.

2 Hatle L, Angelsen BA, Tromsdal A. Non-invasive assessment of aortic stenosis by Doppler ultrasound. $\mathrm{Br}$ Heart $\mathcal{F}$ 1980; 43: 284-92.

3 Hatle L, Angelsen B. Doppler ultrasound in cardiology; physical principles and clinical applications. Philadelphia: Lea and Febiger, 1985: 143-51.

4 Lima CO, Sahn DJ, Valdes-Cruz LM, et al. Noninvasive prediction of transvalvular pressure gradient in patients with pulmonary stenosis by quantitative two-dimensional echocardiographic Doppler studies. Circulation 1983; 67: 866-71.

5 Johnson GL, Kwan OL, Handshoe S, Noonan JA, De Maria AN. Accuracy of combined two-dimensional echocardiography and continuous wave Doppler recordings in the estimation of pressure gradient in right ventricular outlet obstruction. $f \mathrm{Am}$ Coll Cardiol 1984; 3: 1013-8.

6 Kosturakis D, Allen HD, Goldberg SJ, Sahn DJ, Valdes-Cruz LM. Noninvasive quantification of stenotic semilunar valve areas by Doppler echocardiography. 7 Am Coll Cardiol 1984; 3: 1256-62.

7 Goldberg SJ, Allen HD, Marx GR, Flinn CJ. Doppler echocardiography. Philadelphia: Lea and Febiger, 1985: $120-6$.

8 Houston AB, Sheldon CD, Simpson IA, Doig WB, Coleman EN. The severity of pulmonary valve or artery obstruction in children estimated by Doppler ultrasound. Eur Heart $\mathcal{F}$ 1985; 6: 786-90.

9 Hagler DJ, Currie PJ, Seward JB, Fyfe DA, Mair DD, Tajik AJ. Doppler assessment of congenital stenotic cardiac lesions: simultaneous continuous wave Doppler-catheter pressure gradient correlation. In Abstracts, 6th Symposium on Echocardiography, Rotterdam, 1985: 85.

10 Stevenson JG, Kawabori I. Noninvasive determination of pressure gradients in children: two methods employing pulsed Doppler echocardiography. $\mathrm{f}$ Am Coll Cardiol 1984; 3: 179-92.

11 Hatle L, Angelsen B. Doppler ultrasound in cardiology: physical principles and clinical applications. Philadelphia: Lea and Febiger, 1985: 110-24.

12 Hatle L, Angelsen B. Doppler ultrasound in cardiology: physical principles and clinical applications. Philadelphia: Lea and Febiger, 1985: 130-2. 